\title{
Physarum polycephalum: - towards a biological controller
}

\author{
Benjamin Taylor ${ }^{1}$, Andrew Adamatzky ${ }^{2}$, John Greenman $^{3}$ and Ioannis Ieropoulos ${ }^{1, *}$ \\ ${ }^{1}$ Bristol Robotics Laboratory, University of the West of England, Bristol, UK. BS16 1QY \\ ${ }^{2}$ Centre for Unconventional Computing, University of the West of England, Bristol, UK. BS16 1QY \\ ${ }^{3}$ Department of Biological, Biomedical and Analytical Sciences, University of the West of England, Bristol, UK. BS16 1QY \\ *ioannis.ieropoulos@brl.ac.uk
}

\begin{abstract}
Microbial fuels cells (MFCs) are bio-electrochemical transducers that generate energy from the metabolism of electro-active microorganisms. The organism Physarum polycephalum is a slime mould, which has demonstrated many novel and interesting properties in the field of unconventional computation, such as route mapping between nutrient sources, maze solving and nutrient balancing. It is a motile, photosensitive and oxygen-consuming organism, and is known to be symbiotic with some, and antagonistic with other microbial species. In the context of artificial life, the slime mould would provide a biological mechanism (along with the microbial community) for controlling the performance and behaviour of artificial systems (MFCs, robots). In the experiments it was found that $P$. polycephalum did not generate significant amounts of power when inoculated in the anode. However, when $P$. polycephalum was introduced in the cathode of MFCs, a statistically significant difference in power output was observed.
\end{abstract}

\section{Introduction}

Physarum polycephalum has been the subject of numerous studies as a living agent that could be integrated into a biological computer system in the future. The slime mould exhibits some remarkable characteristics that have been exploited by researchers in a plethora of different research areas, including mapping (Tero et al., 2006; Adamatzky and Jones 2010), robotic control (Tsuda et al., 2007), maze solving (Nakagaki et al., 2000) and possibly computing and logic gates (Tsuda et al., 2004; Adamatzky and Schubert, 2014).

$P$. polycephalum can exist in several different forms as it goes through a complex life cycle. It can exist as a spore, a single cell (amoeboid), a food seeking plasmodium (mass of nucleated cells) and as a dormant plasmodium (sclerotium), each stage being dependent on environmental, nutritional and other stress factors.

The most interesting life stage of $P$. polycephalum for biosensing and bioelectronic research currently focuses on the active plasmodium phase. In this mode of growth, $P$. polycephalum is actively motile (has been observed moving up to $10 \mathrm{~mm}$ per hour) and will search for food sources. In addition, it will also lay down protoplasmic tubes to connect any food sources and re-direct nutrients though the protoplasmic tubes by peristalsis (Alim et al., 2013) to the most active regions of the organism. P. polycephalum has been shown to be sensitive to bright light (Ueda et al., 1988) and specific chemical cues (Costello and Adamatzky, 2013), which have been used to control the direction of plasmodium locomotion. These properties of $P$. polycephalum could be beneficial when used with microbial fuel cells and could be exploited in the future as biological switches or behavioural controllers.

Microbial fuel cells (MFCs) are bio-electrochemical systems that exploit the ability of electro-active microorganisms to colonise an electrode, breakdown organic material and donate electrons to the electrode as part of anaerobic respiration. MFCs typically comprise an anode electrode and a cathode electrode separated by a cation exchange membrane (Figure 1). The membrane employed in MFCs is usually non-porous and facilitates the transfer of cations whilst maintaining physical separation between the anode and cathode half-cells. Other membranes have been investigated such as terracotta, earthenware (Winfield et al., 2013) bi-polar membrane and charge mosaic membrane (Rozendal et al., 2008) and have been observed facilitating the transfer of other ions such as $\mathrm{Na}^{+}, \mathrm{K}^{+}$and $\mathrm{NH}_{4}^{+}$across the membrane, but still able maintain a physical separation and potential difference between the half cells. When an electrical load is connected between the two chambers, a current is produced when electrons move through the circuit (load) and protons migrate through the cation exchange membrane to reduce molecular oxygen to water in the cathode.

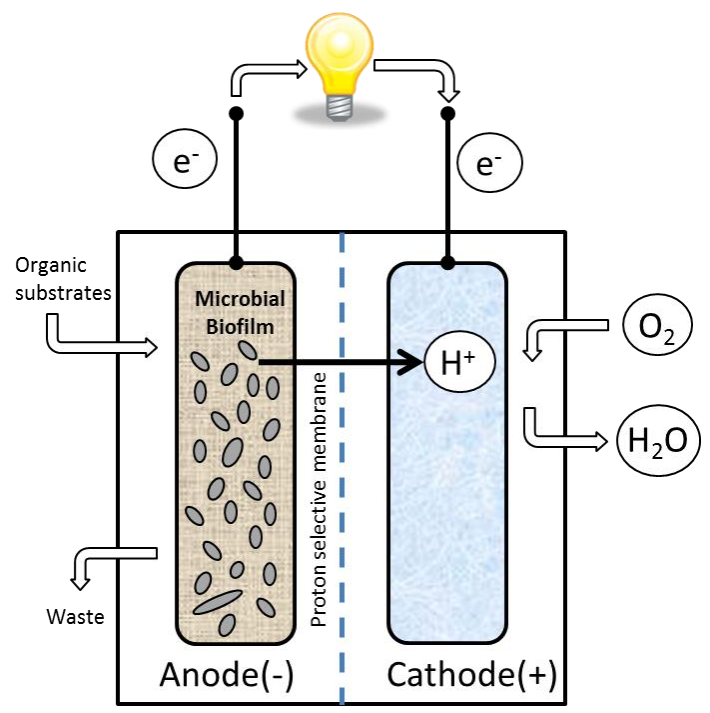


Figure 1. Simplified diagram showing the basic configuration of a MFC. The 2 chambers containing the anode and cathode electrodes are separated by a cation selective membrane, and an external load facilitates electron flow.

The MFC technology is subject to ongoing optimisation exercises by the scientific community, since there are several parameters that can be greatly improved. One of these is the cathode half-cell, which can be of liquid or gaseous form, can involve expensive noble metal catalysts or low cost inert materials, and can also be based on biotic or abiotic operation. Currently, the majority of MFC research has focussed on the treatment and bio-degradation of liquid organic waste and has been used to process sewerage (Liu et al., 2004), urine (Ieropoulos et al., 2013a) and industrial liquid waste such as brewery and food effluent (Oh and Logan 2005; Feng et al., 2008). Examples of practical implementation have been reported on mobile phone recharging (Ieropoulos et al., $2013 \mathrm{~b}$ ) and powering semi-autonomous robotic platforms (Ieropoulos et al., 2005). In addition, MFCs have been used directly as bio-sensors (Kim et al., 1999; Tront et al., 2008) as the current produced from the electro-active community is directly proportional to the growth-limiting organic substrate present in the solution.

The current study introduced for the first time $P$. polycephalum in both the anode and cathode half-cells (separately), in order to investigate the slime-mould's ability to either generate electricity, or affect a performance/behavioural change due to its unique responses to environmental cues. It is envisaged that if implemented onboard robots, then this response may help realise photo- or chemo-tactic behaviour from the artificial agents.

\section{Materials and Methods}

\section{$P$. polycephalum culture conditions}

A plasmodium of $P$. polycephalum was sub-cultured onto sterile $2 \%$ non-nutrient agar (Oxoid Ltd, Basingstoke, UK) on which 3 pea-sized amounts of porridge oats (1:3 w/v oats: hot water [Suma organic porridge oats, Suma, Elland, UK]) were placed approximately $40 \mathrm{~mm}$ apart. All cultures were incubated at room temperature (approximately $21^{\circ} \mathrm{C}$ ) in dark conditions and were sub-cultured weekly.

\section{Experiment 1: $P$. polycephalum anode}

Preparation of the MFC Anode A $270 \mathrm{~cm}^{2}(9 \mathrm{~cm} \mathrm{x} 30 \mathrm{~cm})$ section of carbon veil $\left(20 \mathrm{~g} / \mathrm{m}^{2}\right.$; PRF Composite Materials Ltd, Dorset, UK) was cut to size and $4 \mathrm{~g}$ (dry weight) of porridge oats $(1: 3 \mathrm{w} / \mathrm{v}$ oats: hot water) was spread along the length of the carbon veil. The porridge oats were then folded into the carbon veil and held in place with a nickel-chrome wire. A small pea-sized amount of porridge was spread onto either side of the electrode before being sterilised by autoclaving at $121^{\circ} \mathrm{C}, 15 \mathrm{psi}$ for 15 minutes. This would constitute the anode electrode.
After sterilisation, the electrode was placed into the MFC anode housing and two excised sections of $P$. polycephalum plasmodium were placed onto either side of the anode electrode. The outer housing of the anode was then closed but leaving a small air gap to allow air perfusion.

Preparation of the MFC Cathode A $270 \mathrm{~cm}^{2}(9 \mathrm{~cm} \times 30 \mathrm{~cm})$ section of carbon veil was cut to size, folded and held in place with a nickel-chrome wire. The cathode was then placed into the cathode housing and the compartment filled with deionised water.

Experimental procedure All MFC types were setup in triplicate, with the test MFCs inoculated with $P$. polycephalum (Figure 2) and control MFCs setup in an identical manner, but without the addition of $P$. polycephalum. All experiments were initially left open-circuit for 2.5 days before the addition of a $9.3 \mathrm{kOhm}$ load to each MFC, which was left connected for the duration of the experiment. Continuous monitoring of the MFC voltage was achieved using a Pico log data recorder and software (Pico technology Ltd, Cambridgeshire, UK). Every 3-4 days, the anodes containing activated sludge were enriched with $5 \mathrm{~mL}$ of $1 \%$ tryptone and $0.5 \%$ yeast extract.

\section{Experiment 2: $P$. polycephalum cathode}

Preparation of the MFC Cathode The cathode preparation was identical to the one described above for the MFC anode but with the final electrode being placed in the cathode housing.

Preparation of the MFC Anode A $270 \mathrm{~cm}^{2}(9 \mathrm{~cm} \mathrm{x} 30 \mathrm{~cm})$ section of carbon veil was cut, folded and held in place with a nickel-chrome wire. The anode was placed into the MFC anode housing and inoculated with $25 \mathrm{~mL}$ of activated sludge (Wessex Water Ltd, Keynsham, UK) enriched with $1 \%$ tryptone (Fisher Scientific Ltd, Leicestershire, UK) and $0.5 \%$ yeast extract (Oxoid Ltd, Basingstoke, UK).

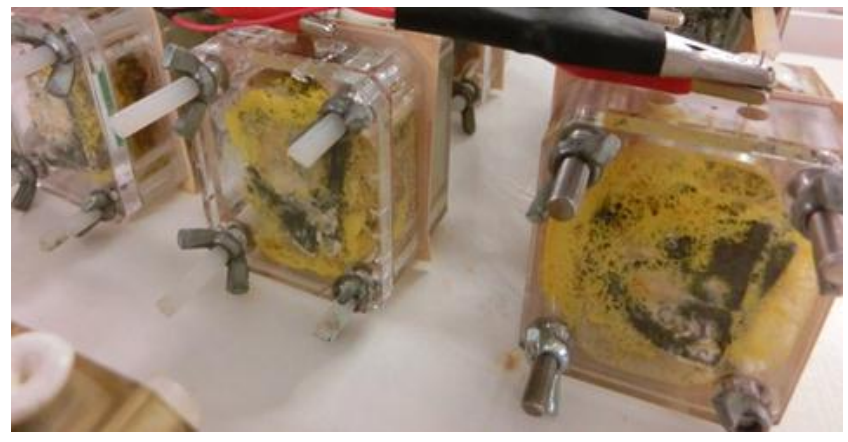

Figure 2. A triplicate of test MFCs with cathode electrodes heavily colonised with yellow $P$. polycephalum.

Power curves Power curves were obtained using an automated computer controlled variable resistor (Degrenne et al., 2012) connected to a Picolog data recorder. Initially the polarisation started at $1 \mathrm{MOhm}$, which was gradually decreased in a stepwise fashion, every 5 minutes, down to $3.74 \mathrm{Ohms}$. The voltage and current were automatically recorded at each resistance value ( $\mathrm{n}=10$ per resistance value). The power $(\mu \mathrm{W})$ was then plotted against current $(\mu \mathrm{A})$. 
Statistical analysis All data were processed using Microsoft Excel and graphs produced using Graphpad, Prism (Graphpad Software Inc, California, USA). All significance testing was conducted using the unpaired $t$-test analysis function in Graphpad Prism.

\section{Results}

\section{Anode $P$. polycephalum experiments.}

A peak open circuit voltage of approximately $0.18 \mathrm{~V}-0.20 \mathrm{~V}$ was observed after 2.5 days for the control and test MFCs, respectively (Figure 3). After the $9.3 \mathrm{kOhm}$ load was connected, the voltage rapidly decreased to $0.04 \mathrm{~V}$ (for both test and control MFCs), which represented a current of approx. $5.4 \mu \mathrm{A}$. The voltage and current continued to decrease until day 5 when the output was effectively zero. Statistical analysis revealed that there was no significant difference between the test and control MFCs.

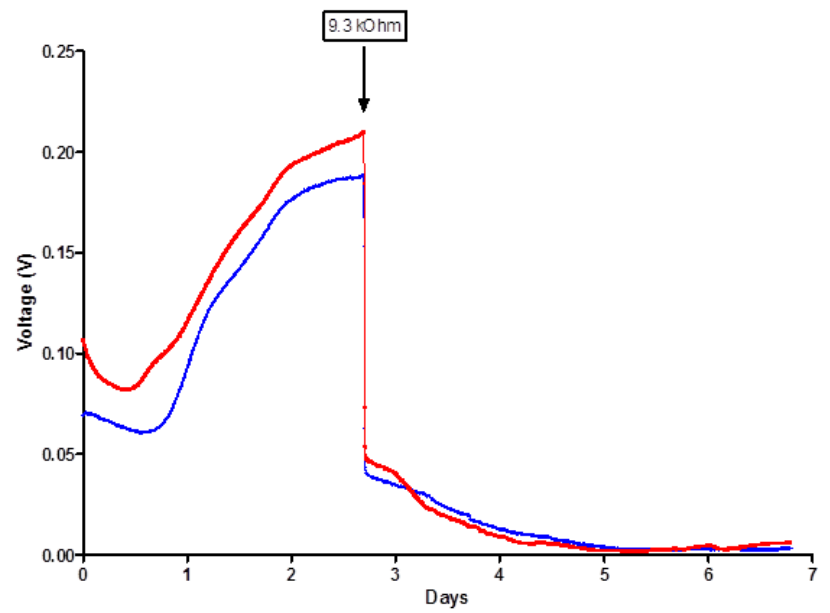

Figure 3. MFC performance with $P$. polycephalum in the anode half-cell. The voltage decreased continuously after the $9.3 \mathrm{kOhm}$ load was applied. Red line $=$ mean for the test MFCs $(n=3)$; blue line = mean for the control MFCs $(n=3)$.

\section{Cathode $P$. polycephalum experiments.}

A peak open circuit voltage of approximately 0.58 and $0.60 \mathrm{~V}$ was observed after 2.5 days for the control and test MFCs, respectively (data not shown). After a $9.3 \mathrm{kOhm}$ load was connected, the voltage rapidly decreased to 0.2 and $0.25 \mathrm{~V}$ for both the control and test MFCs. The initial power output after the load was applied was approx. 4 and $7 \mu \mathrm{W}$, respectively (Figure 4), which increased to 15 and $11 \mu \mathrm{W}$, for the test and control MFCs, respectively, after the sludge anodes were fed with $5 \mathrm{~mL}$ of $1 \%$ tryptone, $0.5 \%$ yeast extract on day 4 (Figure 4). The sludge anodes were fed again on day 7 and generated approximately 10 and $12.5 \mu \mathrm{W}$ (control and test respectively). Statistical analysis revealed that there was a significant difference between the test and control MFCs.

The power curves for the $P$. polycephalum cathode electrodes (Figure 5) indicated a maximum power point of approximately 14.6 and $13.2 \mu \mathrm{W}$ for the test and control MFCs respectively. The resistive load at maximum power was
$1 \mathrm{kOhm}$ for the both the test and control MFCs, which were found to be statistically different $(p<0.05)$ around the maximum peak output between 50 and $80 \mu \mathrm{A}$.

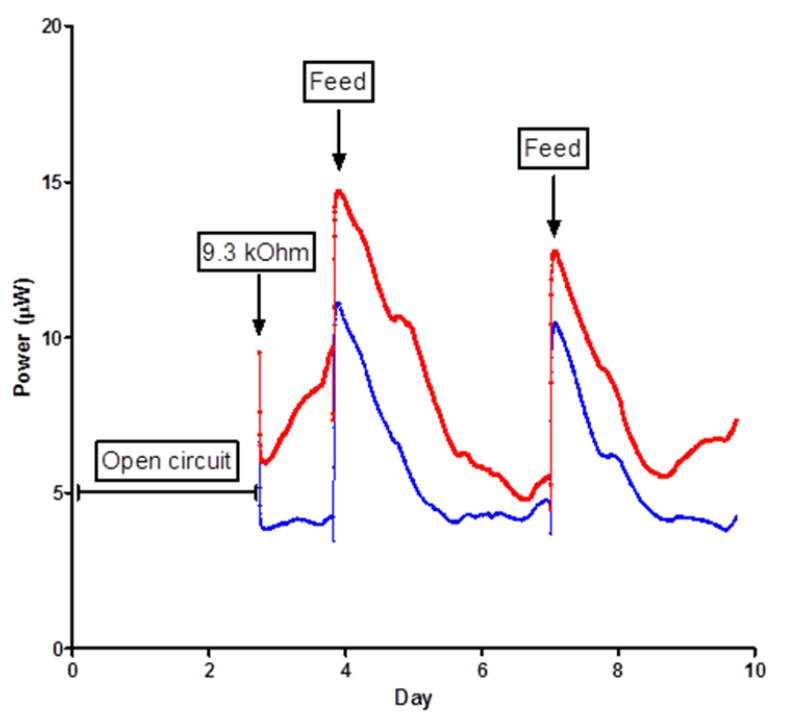

Figure 4. MFC performance with $P$. polycephalum in the cathode. Arrows indicate when the anode chamber containing the activated sludge was fed with $1 \%$ tryptone and $0.5 \%$ yeast extract. There was a significant difference between tests (with $P$. polycephalum) and controls (without $P$. polycephalum). Red line $=$ mean for test MFCs $(n=3)$; blue line $=$ mean for control MFCs $(n=3)$.

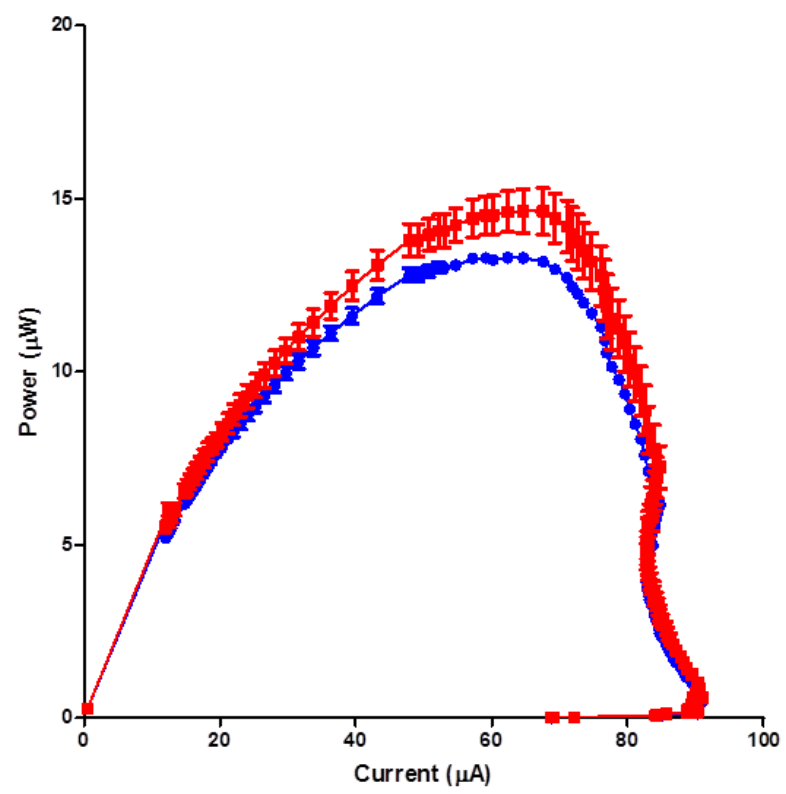

Figure 5. MFC power curves with $P$. polycephalum colonising the cathode. Red line $=$ test $\operatorname{MFCs}(n=3)$; blue line $=$ control MFCs $(n=3)$. Error bars show \pm SEM, $n=10$. 


\section{Discussion}

The aim of this work was to investigate any effects in electrical performance from introducing $P$. polycephalum in MFCs. The initial experiments with the slime mould in the anode showed insignificant power output levels and no statistical differences between test MFCs and control units. This was most likely due to the difference between the aerobic respiration of $P$. polycephalum (being a mitochondria driven process) and other facultative anodophiles, which respire and exchange electrons with the electrode surface. Many species of anodophile are able to directly transfer electrons to the surface of the anode electrode via oxidoreductase pathways present in the outer layers of the bacterial membrane. The exact mechanism of oxidoreductase activity is usually dependant on the organism involved (such as the Mtr pathways for Shewanella species; Coursolle et al., 2010) and it is most likely that $P$. polycephalum lacks any kind of oxidreductase activity in its outer membrane.

However, the open circuit voltage evolution (Figure 3) may be an interesting sensory mechanism for exploitation, in terms of response to environmental conditions. This observation could be the result of the redox potential of dissolved oxygen (approximately $0.8 \mathrm{~V}$ ) in the water cathode; or possibly from secondary metabolic reactions occurring within the porridge oats contained inside the anode electrode.

The improvement in power, when $P$. polycephalum was introduced in the cathode, was unexpected, since it was originally hypothesised that $P$. polycephalum would negatively affect the MFC performance as a result of increased oxygen consumption and demand from the slime mould.

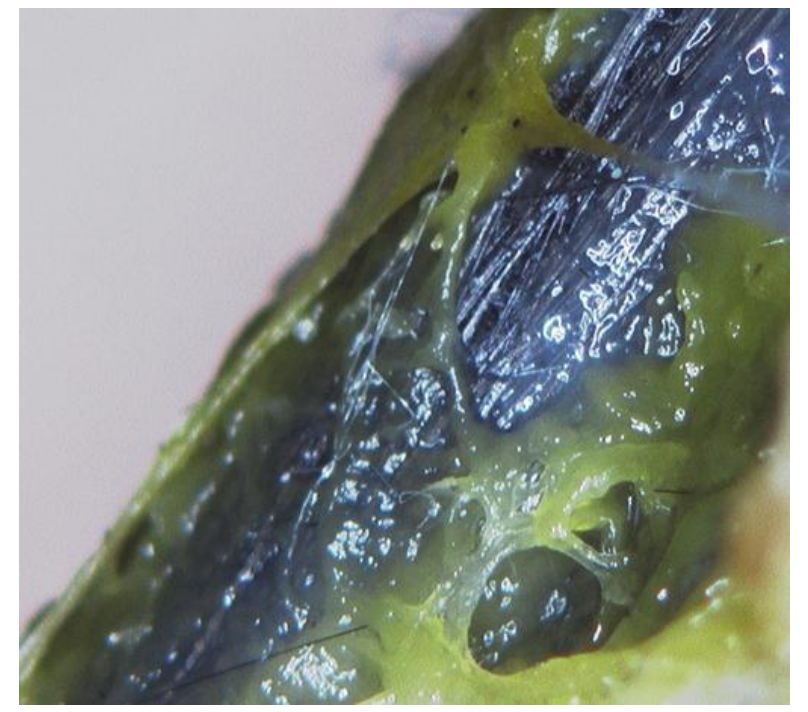

Figure 6. $P$. polycephalum colonising the cathode electrode (50x magnification, Hirox Europe Ltd, Limonest, France). The carbon veil electrode appears glossy and wet due to the presence of mucosa generated by $P$. polycephalum.

As can be seen in Figure 4, there was a significant difference $(p<0.05)$ between the electrodes colonised with $P$. polycephalum and the controls. In addition, a significant difference in peak power output in the ranges of 50 to $80 \mu \mathrm{A}$ was also shown. It is possible to hypothesise that the colonising/locomotive action of $P$. polycephalum increases the contact efficiency between the outer surface of the electrode and the cation exchange membrane. The increased contact facilitates the proton exchange efficiency between the anodic and cathodic compartments of the MFC, and results in the increased power output observed. The protoplasmic tubes of $P$. polycephalum contain relatively large proportions of water and dissolved oxygen in addition to the nutrients being transported around the plasmodia as it forages for food sources (Dussutour et al., 2010). These protoplasmic tubes could easily be observed covering the surface of the electrode (Figure 6) as they transport the breakdown products of the porridge (hydrolysed oligo- and monosaccharides) deposited inside the carbon veil and it is this transport of water and oxygen around the electrode that may have improved the cathode performance.

The power curves were generated from the polarisation experiment, to identify the maximum power point from the MFCs. The polarisation run covered a range of $1 \mathrm{MOhm}$ to $3.740 h m s$ for the external load. It was found that the value, which generated maximum power, was $1 \mathrm{kOhm}$ for both the test and control MFCs.

It is important to note that these experiments were conducted in batch mode, which resulted in periodic substrate replenishment; hence the peaks in Fig.4. A way to remove this requirement would be to operate under continuous flow using a peristaltic pump supplying media from a large vessel.

The immediate next steps in this line of experiments will be to capture some interesting behavioural patterns from $P$. polycephalum, for example response to light, inside the MFC cathodes, and demonstrate this as a direct electrical output. This may allow the direct control of external devices, based on the MFC signal. This particular slime mould is adverse to humid conditions. These are interesting traits, which if exploited appropriately at a higher level of complexity, for example being an integral part of a robot such as EcoBot (Ieropoulos et al. 2010), could result in a new form of biological 'processor' for an artificial agent.

\section{Conclusions}

A small but statistically significant difference was observed for MFC power output for $P$. polycephalum colonised cathodes (over control units) and this difference could be exploited in the future to control power output from microbial fuel cells. Future research using this combination of MFCs and $P$. polycephalum will aim to directly control a mechanical or electrical device.

\section{Acknowledgements}

The authors would like to thank the European Commission for funding this work under the Seventh Framework Programme (FP7) "Physarum Chip: Growing Computers from Slime Mould". Project reference 316366. Website http://www.phychip.eu 


\section{References}

Adamatzky A and Jones J (2010) Road planning with slime mould: If Physarum built motorways it would route M6/M74 through Newcastle. Int. J. Bifurcation and Chaos. 20 (10) 3065-84.

Adamatzky A and Schubert T (2014) Slime mold microfluidic logical gates. Materials Today. 17 (2) 86-91.

Alim K, Amselema G, Peaudecerf F, Brenner M P and Pringle A (2013) Random network peristalsis in Physarum polycephalum organizes fluid flows across an individual. PNAS: 110 (33).

Costello B de L and Adamatzky A (2013) Assessing the chemotaxis behavior of Physarum polycephalum to a range of simple volatile organic chemicals. Commun. Integr. Biol. 6 (5).

Coursolle D, Baron D B, Bond D R and Gralnic J A (2010) The Mtr respiratory pathway is essential for reducing flavins and electrodes in Shewanella oneidensis. J. Bacteriol. 192 (2) 46774

Degrenne N, Buret F, Allard B, Bevilacqua P (2012) Electrical energy generation from a large number of microbial fuel cells operating at maximum power point electrical load. J. Power Sources (205) 188-93

Dussutour A, Latty T, Beekman M, Simpson J (2010) Amoeboid organism solves complex nutritional challenges. PNAS. 107 (10) 4607-11

Feng Y, Wang X and Logan B (2008) Brewery wastewater treatment using air-cathode microbial fuel cells. Appl. Microbiol. Biotechnol. 78(5) 873-880.

Ieropoulos I, Greenman J, Lewis D and Knoop O (2013a) Energy production and sanitation improvement using microbial fuel cells. J. Water, Sanitation and Hygiene for Development. doi:10.2166/washdev.2013.117.

Ieropoulos, I., Greenman, J., Melhuish, C and Horsfield, I. (2010) EcoBot-III: a robot with guts, In Fellermann, H., Dörr, M., Hanczyc, M., et al., editors, Artificial Life XII, pages 733-740. MIT Press, Cambridge, MA.

Ieropoulos I, Melhuish C, Greenman J and Horsfield I (2005) EcoBot-II: An artificial agent with a natural metabolism. Int. J. Advanced Robotic Systems. 2 (4) 295-300.

Ieropoulos I, Ledezma P, Stinchcombe A, Papaharalabos G, Melhuish C and Greenman J (2013b) Waste to real energy: the first MFC powered mobile phone. Phys. Chem. 15 15312-16.

Kim H J, Moon S H, In S C and Byung H K (1999) A microbial fuel cell type lactate biosensor using a metal-reducing bacterium, Shewanella putrefaciens. J. Microbiol. Biotechnol. 9 (3) 365-7.

Liu H, Ramnarayanan R and Logan B (2004) Production of Electricity during Wastewater Treatment Using a Single Chamber Microbial Fuel Cell. Environ. Sci. Technol. 38 (7) 2281-5.

Nakagaki T, Yamada H and Tóth A (2000) Intelligence: Mazesolving by an amoeboid organism. Nature. doi: $10.1038 / 35035159$.

Oh S and Logan B (2005) Hydrogen and electricity production from a food processing wastewater using fermentation and microbial fuel cell technologies. Water Research. 39 (19) 4673-82.

Rozendal R A, Sleutels, T H, Hamelers H V and Buisman C J (2008) Effect of the type of ion exchange membrane on performance, ion transport, and $\mathrm{pH}$ in biocatalyzed electrolysis of wastewater. Water Sci. Tech. 57 (11) 1757-62

Tero A, Kobayashia R and Nakagakib T (2006) Physarum solver: A biologically inspired method of road-network navigation. Physica A. 363 (1) 115-119.

Tront J M, Fortner J D, Plötze M, Hughes J B and Puzrin A M (2008) Microbial fuel cell biosensor for in situ assessment of microbial activity. Biosens. Bioelectron. 24 (4) 586-90.

Tsuda S, Zaunerb K P and Gunjia Y P (2007) Robot control with biological cells. BioSystems. 87 215-223.

Tsuda S, Aono M and Gunjia Y P (2004) Robust and emergent Physarum logical-computing. BioSystems. 73 45-55.

Ueda T, Mori Y, Nakagaki T and Kobatake Y (1988) Action spectra for superoxide genera and UV and visible light photoaviodance in plasmodia of Physarum polycephalum. Photochem. Photobiol. 48 (5) 705-9.

Winfield J, Greenman J, Huson D and Ieropoulos I (2013) Comparing terracotta and earthenware for multiple functionalities in microbial fuel cells. Bioproc Biosyst Eng 36 (12) 1913-21. 